\title{
Learning from experience: A simple effective protocol to test footwear prescriptions for the Diabetic foot by using the Pedar system
}

\author{
Claudia Giacomozzi ${ }^{1}$, Luigi Uccioli ${ }^{2}$ \\ ${ }^{1}$ Department of Technology and Health, Italian National Institute of Health, Rome, Italy \\ ${ }^{2}$ Department of Internal Medicine, Tor Vergata University Hospital, Rome, Italy \\ Email: c_giacomozzi@yahoo.com
}

Received 10 March 2013; revised 13 April 2013; accepted 14 May 2013

Copyright (C 2013 Claudia Giacomozzi, Luigi Uccioli. This is an open access article distributed under the Creative Commons Attribution License, which permits unrestricted use, distribution, and reproduction in any medium, provided the original work is properly cited.

\begin{abstract}
Adherence of patients to treatment and appropriateness of prescribed footwear are mandatory for successful prevention of Diabetic foot ulceration. In a Public Healthcare Service, footwear approval is under the responsibility of the clinician. In some cases kinetic measurements are taken inside the shoes to support the clinical decision; however, an overall agreement is still missing with respect to a simple, reliable and effective test protocol based on proper threshold values. Authors' past experiences, specific needs of the Diabetic outpatient service, and suggestions from valuable literature led to an instrumental test protocol based on reference peak pressure (PP) thresholds and gait line (GL) specific for each level of risk. Permission was obtained for one preliminary validation session at the Authors' outpatient service, during which 11 patients at high/very high risk of ulceration were examined-9 for testing new footwear, 2 for monitoring 12-month-old footwear. The protocol was well accepted by patients and operators, fast and easy to be used. Based on the instrumental, clinical and visual inspection data; 4 new footwear did not pass the test; 5 passed the test but integration or minor changes were requested; the 2 old footwear were found no more effective. As a first positive feedback, after 4 months of wearing the modified prescriptions none of the patients had developed plantar ulcers or signs of tissue damage. Successive data processing proved that in all patients altered PPs and deviated GLs did correlate with alterations of other kinetic parameters. The protocol, which is proved to be sensitive and valuable for the Diabetic foot care, may have a general validity; the delivered specific PP
\end{abstract}

thresholds and reference data, instead, can be reliably used if the test is performed with a calibrated Pedar Insole System and with the wide Insoles in the range UW-YW (EU size 36 - 45).

Keywords: Diabetic Foot; Ulcer Prevention; Footwear; Instrumental Test; Plantar Pressure; Gait Line

\section{INTRODUCTION}

The process of care of the Diabetic neuropathic foot is quite complex. Some of the fundamental steps of the process, which aims at preventing ulcer formation or recurrence, have been deeply investigated and have already reached a satisfactory level of maturity and effectiveness at least in developed countries; some others are still debated. Education and training of healthcare operators, patients and caregivers, for example, are nowadays highly quailfied and effectively delivered on a territorial basis; to support this action, official Guidelines for home care of the Diabetic foot, personal foot inspection, proper selection of shoes and insoles are widely disseminated world-wide [1]. As a second fundamental step of the process, foot screening and identification of the class of risk are well established too: typically, a Diabetic foot is classified as a low-medium risk foot, a high risk foot or a very high risk foot, and footwear prescription-shoes and insoles—is done accordingly to agreed and well established recommendations [2,3]. At least two further steps are fundamental in the whole process, which is still in a consolidation phase and whose effectiveness is yet under investigation: 1) a standardised, objective approach for testing and monitoring footwear prescription, and 2) an effective approach to increase adherence of Diabetic patients to wear the prescribed footwear as 
much as possible. As for the second point, a recent valuable objective study [4] demonstrated that adherence is still too low to render any preventive action effective in avoiding ulceration or recurrence of ulceration. However, while working to successfully overcome such crucial issue, a proper testing of footwear prescription is equally relevant and worth to be investigated, since it implies benefits at different levels: 1) clinically, it allows the effective off-loading of foot areas at risk of ulceration, keeping the areas healthy or healed for longer time; 2) at an economical level, the correct footwear prescription and its replacement at the right moment its effectiveness is lost may entail the optimisation of healthcare resources; 3) from the quality perception point of view, a more effective footwear might entail a higher patient's compliance and motivation and, hopefully, a greater adherence to preventive treatments.

The present study is focussed on the proposal of a standardised, objective approach for measuring the performance of footwear prescriptions during their test at an outpatient service for Diabetic foot, so as to deliver an objective instrumental support to the clinical decision. Several studies, in fact, have already been published on the topic, where an instrumental approach is implemented and kinetic measurements are taken inside the shoes to test insoles, shoes or both [5-9]; however, an overall agreement is still missing with respect to a replicable, simple, reliable test protocol which, based on few parameters and proper threshold values, may be effective and prompt to be used by clinicians to sustain or rebut their decision.

The rationale for the new approach hereby proposed relies on multiple factors and experiences, and of course on evidence and suggestions from the literature.

As a starting point, elevated plantar pressures and significant alterations of plantar pressure curve along the entire roll-off process of the foot are associated with Diabetic neuropathic barefoot gait, the alterations being more evident for more severe stages of the disease [10, 11]. Second, and equally important, is the consolidated evidence that high values of peak plantar pressures and poor fitting of footwear are recognized as important risk factors in the Diabetic foot ulceration process [12,13]. Thus, focus should be on the accurate measurement of peak plantar pressure and on the identification of proper risk threshold. A milestone of the literature in this sense is represented by the 2009 paper from Cavanagh and coauthors [14]. They started from a clinical point of view, on the basis of the following hypothesis: Diabetic neuropathic patients who had prior foot ulcers that remained healed during the observation period (90 days) were likely walking with proper footwear so as to avoid any risky plantar pressure. In their cohort study on 40 patients they thus measured insole plantar pressures dur- ing regular walking by means of a Pedar Insole System. Notwithstanding the inter-patient variability, they suggested to adopt the plantar pressure threshold of $200 \mathrm{kPa}$ in correspondence with previous ulcer sites.

However, as Prof Cavanagh himself commented in a 2011 interview to the specialized on-line magazine LER [15], the level of pressure at which tissue damage occurs in a Diabetic neuropathic foot is yet to be defined. Such an uncertainty has for sure to be ascribed not only to the variability of clinical and treatment conditions, but also to the great variability in the performance of in-shoe plantar pressure measurement systems. Available devices, in fact, perform differently one from the others due to several factors, e.g. sensor technology and dimension, pressure range, mechanical assembly and cover, sampling rate, pressure range $[9,16]$. Furthermore, different shoe and insole material and shape, as well as the implemented protocol and dedicated data processing, greatly influence the device pressure outcome [17-19].

Preliminary studies conducted at the Authors' labs with a Pedar Insole System and under measurement conditions comparable with [14] showed that, in some cases, the suggested threshold of $200 \mathrm{kPa}$ might be not enough sensitive for the Authors' outpatients, thus not helping to discriminate among the proposed footwear clinical effects. In some cases, for example, other kinetic parameters were found altered while peak pressure remained under the suggested threshold. Besides this, the gait line deviations were found to be extremely relevant, too, for the overall footwear performance assessment of Diabetic patients, in agreement with reported gait line deviation associated with Diabetic neuropathy during barefoot walking [20].

Thus, while taking into account the suggested threshold [14], a different hypothesis was formulated and a different approach followed in the present study. Basically, healthy volunteers were recruited who matched as much as possible the "typical" patient of the Authors' outpatient Diabetic foot service-as for age, BMI, walking speed-but who had no calluses at all under their feet. This latter feature was interpreted as a sign of a proper mechanical loading for each individual. A reference database was then created by averaging data from the healthy volunteers while walking in an adequate, modified semi-rigid shoe. Reference peak pressures were identified for 4 foot subareas-rearfoot, midfoot, forefoot and toes-and some correction factors were applied to take into account for the different risk level, and the different foot sizes which, in the Pedar Insole System, entail different areas of corresponding sensors (all relevant details of the study are reported and explained in the Methods Section).

Permission was obtained at the Authors' Hospital for a preliminary validation session during one day at the out- 
patient Diabetic foot service. The protocol, applied to 11 Diabetic patients, was well accepted by patients and operators, and resulted fast and easy to be used and interpreted. Footwear test results, obtained as an integration of instrumental, clinical and visual inspection data, are briefly reported and commented in the paper. Encouragingly, 4 months after the preliminary validation session, none of the patients had developed plantar ulcers or showed signs of tissue damage. Thus, the protocol was judged not only feasible and well accepted, but also safe and helpful in the process of care of the Diabetic neuropathic foot.

Aiming at further validating and improving this approach, all indications, thresholds and reference data and details are reported in the paper to allow everyone the correct implementation of the test protocol under comparable conditions. Worth to remember, while the suggested methodology is of general applicability, the used pressure thresholds and reference gait line are to be considered valid whenever the footwear test is performed by using a calibrated Pedar Insole System, and measurements are taken by using the wide model of the Pedar Insoles in the range UW-YW (i.e. EU size 36 - 45).

\section{MATERIALS AND METHODS}

\subsection{Background and Rationale}

The background issues hereby summarized formed the basis and the rationale for the choices done in the study to build, implement and use the proposed footwear test protocol.

- Instantaneous peak pressure (PP) may be considered as one of the most important parameter to be used as a risk indicator when dealing with Diabetic foot at risk of ulceration [10]. Furthermore, previous studies [20] showed that significant changes also occur in the trajectory of the center of pressure (COP), here indicated as gait line (GL). Besides these two parameters, few more quantities may be precious in a clinical scenario for the identification of abnormalities of foot-ground interaction during gait, e.g. maximum force normalized to body weight (Max F), force-time integral normalized to body weight (FTI), pressuretime integral (PTI), stance duration (Stance) [19].

- The insole pressure measurement system must be accurate and reliable; being used for measurements addressed to medical purposes, the whole systemhardware and software-should be marketed as a Medical Device with Measuring Function [17,18]. Literature reports about high variability in the performance and outcomes of the available pressure measurement systems in general [16] and, more specifically, of wearable systems [9]. Discussing these issues is beyond the scope of this paper; they are however worth to be kept in mind since each and every proposed protocol should be considered valid only under very well defined measurement conditions. Besides the accuracy, the elasticity/deformation and the thickness of the measurement systems, also the active surface of the pressure sensors does play a special role in detecting peak pressures as close as possible to the expected ones.

- Due to the high intra-variability of each measurements system responses when working with shoes and insoles different as for both material and shape, a reference database might be usefully obtained by using only one kind of footwear, unless a huge number of measurements can be taken under all the expected conditions. Barefoot measurements cannot be considered comparable with in-shoe measurements and should not be used for this specific purpose.

- When recruiting healthy volunteers to build reference databases, attention should be paid to select them, on average, as much comparable as possible with the expected patients as for age, BMI and walking speed, the last two directly interfering with most of the suggested parameters, especially with FTI and PTI.

- When defining an assessment protocol at an outpatient service, it is mandatory that patients' dressing and training, data acquisition, data processing and data interpretation are as clear, repeatable, easy and fast as possible, requirements which are peculiar of the specific scenario and which are not necessarily fulfilled/foregone in a research setting.

- Again on the last point: one of the fastest outcomes of most pressure measurement systems is the maximum peak pressure map, i.e., for each activated sensor, the maximum value of pressure the sensor has recorded during the entire acquisition period. Attention should be paid not to use this outcome for quick data interpretation: as well known, in fact, a certain number of steps - at least twelve-is recommended for reliable measurements [21], and the reliable value of peak pressure for each individual sensor should be calculated by averaging all peak pressure values recorded by that specific sensor in the different steps; reasonably, the averaged peak pressure is lower-and in some cases may be much lower-than the maximum peak pressure.

\subsection{Measurement Equipment, Hardware and Software}

The Pedar-X Insole Measurement System (novel GmbH, Munich, Germany) has been used, which is Certified as a Medical Device in class I with Measuring Function according to the EU Directives [18]. The system is based on insoles of 99 capacitive sensors each, with variable 
sensor active surface (mean sensor surface is around 1\% of insole total surface in wide model insoles), embedded in a $1.5 \mathrm{~mm}$ soft interface (overall thickness $3.4 \mathrm{~mm}$ ). Due to its features, pressure range for in-shoe measurements is reasonably calibrated in the range $15-600 \mathrm{kPa}$. When correctly maintained and used, system has accuracy $<2 \% \mathrm{FS}$, pressure resolution $2.5 \mathrm{kPa}$, hysteresis $<7 \%$. Sampling frequency can go up to 100 samples/s but for the purpose of this study it has been limited to 50 samples/s.

Insoles are delivered in different sizes as for both width and length. Only wide insoles-identified as W series-have been used in the study, from EU size 36 to 45, each insole covering two sizes (Pedar codes from UW to YW): it was in fact hypothesized that this range does cover most of the expected Diabetic patients' foot size at the Authors' Hospital. In the used insoles, sensor active surface increases from the smallest (UW) to the biggest (YW) in a way that maintains sensor shape invariant; sensor surface increase is equal to $11 \%(\mathrm{VW})$, $20 \%$ (WW), 28\% (XW) and 35\% (YW) with respect to UW sensors. Since sensors are not rigid, it is reasonable to hypothesize that a localized load acting on a surface smaller than the sensor surface is smoothed and underestimated, the effect being more evident with wider sensor surfaces. Thus, to render measurements from different insoles comparable, a normalization factor has been introduced by considering the UW insole-the one with the smallest sensors-as the reference.

To acquire reference data to be reasonably compared with patients' outcomes, a post-surgery semi-rigid shoe was chosen (Terapes by Podartis, Italy): it is wide enough to accommodate the wide Pedar insoles and most foot types of the expected patients; it is rigid enough to partly prevent the physiological flexibility of the foot during the roll-over process, thus acting like most of semi-rigid or rigid prescribed shoes; it has no heel or internal support which may further mask the foot-ankle behaviour during gait. Before using it, the shoe has been modified by smoothing and partly removing the rubber sole. Figure 1 shows the hereby described equipment.

Two software packages have been used which are dededicated by the Company to the Pedar System: 1) the Pedar Acquisition tool allows for data acquisition and

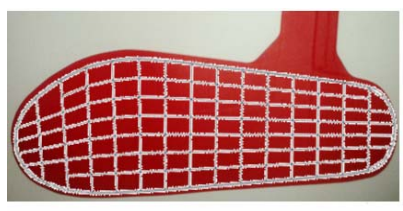

(a)

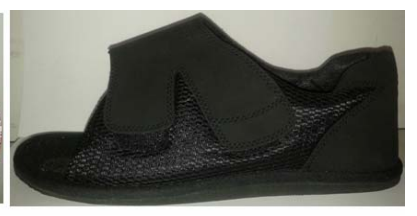

(b)
Figure 1. (a) Pedar-X System wide insole with superimposition of sensor map; (b) Smoothed semirigid Terapes shoe used to collect reference data. some preliminary processing like the identification of insole subareas of interest. As said before, it is highly recommended not to work on the maximum peak pressure maps delivered by this tool right at the end of the acquisition trial, since they represent an overestimation of the averaged peak pressure values: some preliminary tests on 20 feet showed that differences between maximum peak pressures and averaged peak pressures during walking were up to $110 \mathrm{kPa}$ (78 $\pm 34.5 \mathrm{kPa})$; 2) the Pedar Step Analysis tool allows for the fast processing of all the valid steps for each foot, and delivers the averaged data necessary for supporting the clinical decision during the footwear test-i.e. PP and GL-and for further research (Max F, PTI, FTI, Stance).

\subsection{Identification of Reference Data and Thresholds}

Reference data were collected from 20 healthy volunteers with no present or past lower limb injuries, orthopaedic pathologies, or neurological impairments, selected among those healthy volunteers who best resembled the expected Diabetic patients as for age ( $>55$ years), BMI $\left(>25 \mathrm{~kg} / \mathrm{m}^{2}\right)$, long stance phase (>700 ms). Special attention was paid in including only volunteers without calluses under the sole of the foot: absence of calluses was in fact assumed as the external proof that the overall mechanical stress due to everyday motor activities was below any tissue alteration/damage for that specific foot. As a consequence, it was assumed that mechanical stress during walking was below risk threshold, too. Volunteers were equipped with the Pedar Insole System and the modified Terapes shoes; after they familiarized with the whole equipment and the protocol, at least 20 regular steps per foot were acquired during their level walking along a straight corridor close to the outpatient Diabetic foot service room.

Proper masks were created for the insoles to detect four major areas of the foot: rearfoot (RF), midfoot (MF), forefoot (FF), and toes (TO). The masks, which are slightly different from the standard ones proposed by most systems, were based on Authors' previous experiences on footprint masking on the basis of anatomical references [22]. Two masks were defined for each insole to take into account for the two different foot sizes each insole is used for-basically, for the smaller size mask the boundaries were moved one sensor back with respect to the bigger one. Figure 2 shows the four foot regions identified on the insole, with the standard anatomical foot superimposed to the insole frame. The Figure is referred to the Pedar wide VW Insole; however, regions are equally identified in the whole range of used insoles (UW-YW).

Once acquired, force data within each subarea were normalized with respect to body weight, stance data were 


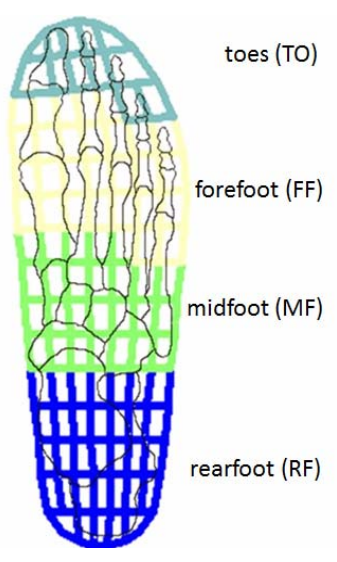

Figure 2. Identification of four foot regions on a Pedar wide insole VW.

normalized with respect to the whole stance duration; successively, all data were averaged within each healthy volunteer, and among all volunteers, keeping data of the two feet separate. Since sensor configuration is the same for all the used insoles, averaged gait line (GL) for each volunteer was mapped on a standard wide insole, and GLs successively averaged over all volunteers.

With respect to peak pressure values only, further processing was performed to obtain pressure thresholds specific for foot size and for Diabetic risk level defined from 0 (lowest) to 3 (highest) as in [3]. The following steps were thus performed, all addressed to define PP thresholds in the most cautious way:

- Since reference PP data from healthy volunteers were mostly referred to VW Pedar insoles (18 volunteers out of 20), the mean PP values were assumed as the maximum PP acceptable values to be associated with VW Pedar insoles and with the low-medium risk level (0 - 1); to be more cautious, the lowest PP between right and left foot was adopted as a maximum threshold, and the 5\% - 7\% variation upwards neglected.

- PP threshold values were progressively reduced, for insoles WW, XW and YW, on the basis of the corresponding \% increase of sensor area with respect to VW sensors, and increased for UW insoles.

- For a specific $n \mathrm{~W}$ insole ( $n=\mathrm{U}$ to Y), the PP threshold values were further reduced of $10 \%$ in case of high risk (risk level 2) and of $20 \%$ in case of very high risk (risk level 3). Main reason for the established percentage relies in the PP variability found within the healthy group, which suggested that any evident, clinically relevant difference of PP between two groups should be greater than $10 \%$.

- The last step consisted in fixing the midfoot PP threshold at $60 \mathrm{kPa}$ for both risk level 2 and 3 independently on the foot size: this arbitrary threshold, in some cases lower than the calculated one, was suggested by Authors' previous clinical experience, by preliminary measurements, and by the observation that the Diabetic foot skin in the midfoot area is often especially fragile.

The last step of this long and complex preliminary phase consisted in the preparation of 30 types of paper sheets to be delivered to the footwear test operator: 10 sheets were in fact prepared for each of the three risk levels, because 2 sheets- 1 for the smaller and 1 for the bigger size mask-are necessary for each of the 5 Pedar wide insole sizes.

\subsection{Protocol}

The present protocol was only applied once at the Authors' Hospital as a preliminary validation session. However, it is here reported in the form of a routine activity at the Hospital, as it will hopefully become soon.

When a patient is accepted at the Authors' outpatient service for Diabetic foot, he/she first receives the clinical examination and is then introduced to the testing room where he is informed on the instrumental assessment and asked to sign the informed consent. Once equipped with the measurement system and the reference shoe (i.e. the modified Terapes shoes), he/she receives further instructions. Few minutes are dedicated to get him/her familiar with the equipment, the task and the shoes. Meanwhile, the operator selects the proper pre-printed paper sheet for reporting the main test results, i.e. he/she takes the pre-printed paper with PP thresholds associated with the Pedar insole size used for the specific patient and with his/her level of risk. The patient is then acquired while walking at his/her self-selected speed along the adjacent corridor until at least 20 valid footprints are acquired per foot. The whole process is repeated with the patient wearing the prescribed footwear under test. While the patient is assisted for removing the equipment, the data related to the prescribed footwear are associated with the proper insole masks and passed to the Step Analysis tool for the requested automatic processing. Mean PP values and averaged GL are made immediately available on the screen and the operator can take notes on the pre-selected paper sheet. Basically, each sheet contains a pair of left and right insoles with PP threshold values for the four foot regions, and a black trace of the reference GL; the operator is asked to fill the paper sheet with red circles to mark areas of over-threshold PPs, and black crosses to indicate deviations from reference GL. A white box fornotes is available on the right side of the sheet, to add comments and suggestions for the clinician who has to approve or reject the footwear. While the clinician takes the decision, all the patient's acquired and processed data are stored for further analysis and for research. 


\subsection{Preliminary Validation Session}

The Authors' Hospital gave permission for a first preliminary validation session which was performed on October $25^{\text {th }}, 2012$. The protocol was applied to 11 Diabetic patients classified as high (class 2, 6 patients) and very high (class 3, 5 patients) risk patients; 9 patients were testing new footwear, 2 were monitoring the performance of 12-months-old footwear.

As already explained, PP and GL values referred to the prescribed footwear were delivered promptly and used by the clinician to support the final decision.

The whole set of collected data, from both prescribed footwear and reference shoes, were further processed and analysed to investigate feasibility, safety, appropriateness and usefulness of the proposed instrumental test. More specifically, prescribed footwear data were investigated to verify whether or not the abnormalities highlighted by the established PP thresholds and the reference GL were associated with concurrent alterations of the other relevant baropodometric parameters. Furthermore, data associated with the reference shoes were examined to verify whether the suggested overall equipment is suitable to highlight abnormalities of gait performance in Diabetic neuropathic patients with respect to comparable healthy volunteers and, even more important, whether it is suitable for detecting changes in gait performance due to prescribed footwear when compared with reference semi-rigid shoes.

\section{RESULTS}

\subsection{Reference Data and Thresholds}

Anthropometric data from the 20 healthy volunteers are reported in Table 1 together with stance duration, averaged over the 40 feet of the 20 volunteers.

Reference baropodometric data for the total foot (TF) and for the four foot regions are reported in Table 2. They have been obtained by averaging the results from the 40 feet, i.e. left and right foot have been treated separately for each volunteer. Data are referred to healthy volunteers walking slowly with reference semi-rigid shoes (modified Terapes); it is worth to note that their speed was natural, not artificially slowed down. Most of them (18 out of 20) used the VW Insoles, the remaining

Table 1. Healthy volunteers main data.

\begin{tabular}{cccccc}
\hline & $\begin{array}{c}\text { Age } \\
(\text { years })\end{array}$ & $\begin{array}{c}\text { Height } \\
(\mathrm{cm})\end{array}$ & $\begin{array}{c}\text { Body mass } \\
(\mathrm{kg})\end{array}$ & $\begin{array}{c}\text { BMI } \\
\left(\mathrm{kg} / \mathrm{m}^{2}\right)\end{array}$ & $\begin{array}{c}\text { Stance } \\
(\mathrm{ms})\end{array}$ \\
\hline mean & 67 & 165 & 75 & 27.3 & 821 \\
sd & 7.9 & 8.8 & 8.1 & 3.5 & 101.8 \\
$\mathrm{n}$ & 20 & 20 & 20 & 20 & 40 \\
\hline
\end{tabular}

two volunteers used the WW and the XW insoles respectively.

Figure 3 indicates the reference PP values, kept separate for the left and the right foot, and GL for healthy volunteers. As expected, GL was found to be symmetrical, with the averaged GL variation always remaining within each filled sensor.

As explained in the Methods Section, PP values were conservatively reduced by using reasonable factors to take into account for the variable sensor surface across

Table 2. Healthy volunteers main baropodometric data.

\begin{tabular}{|c|c|c|c|c|c|}
\hline & $\mathrm{TF}$ & $\mathrm{RF}$ & MF & $\mathrm{FF}$ & $\mathrm{TO}$ \\
\hline \multicolumn{6}{|l|}{ Max F } \\
\hline mean $(\% \mathrm{~N})$ & 106 & 66 & 22 & 85 & 16 \\
\hline sd (\%) & 1.6 & 8.2 & 7.7 & 12.1 & 22.6 \\
\hline \multicolumn{6}{|l|}{ PP } \\
\hline mean $(\mathrm{kPa})$ & 248 & 214 & 91 & 249 & 149 \\
\hline sd (\%) & 9.3 & 8.7 & 10.7 & 14.5 & 20.3 \\
\hline \multicolumn{6}{|l|}{ FTI } \\
\hline mean $\left(\% N^{*} s\right)$ & 68 & 24 & 9 & 32 & 4 \\
\hline sd (\%) & 7.5 & 20.8 & 15.7 & 10.8 & 23.4 \\
\hline \multicolumn{6}{|l|}{ PTI } \\
\hline mean $\left(\mathrm{kPa}^{*} \mathrm{~s}\right)$ & 140 & 81 & 45 & 88 & 37 \\
\hline sd (\%) & 9.9 & 18.9 & 18.0 & 10.5 & 18.3 \\
\hline \multicolumn{6}{|l|}{ Stance } \\
\hline mean (ms) & 821 & 591 & 677 & 753 & 559 \\
\hline sd (\%) & 11.4 & 13.1 & 10.2 & 7.9 & 15.3 \\
\hline mean (\%) & 100 & 72 & 82 & 92 & 68 \\
\hline
\end{tabular}

PP REFERENCE VALUES

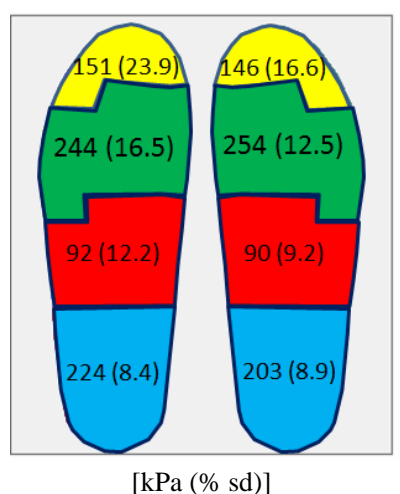

(a)



(b)
Figure 3. PP reference values (a) and reference GL (b) for the four identified foot regions. Data are referred to the 20 healthy volunteers while walking with the modified semi-rigid shoes. 
the used range of insole sizes, and to establish more cautious thresholds for higher levels of risk of ulceration.

Furthermore, as already explained, PP threshold for the midfoot area was fixed to $60 \mathrm{kPa}$ for risk level 2 and 3 independently on the foot size.

The obtained reference PP thresholds are reported in Table 3.

\subsection{Preliminary Footwear Test Results}

During the preliminary validation session on October 2012, the protocol was applied to 11 Diabetic outpatients who had been referred to the Authors' Hospital for monitoring the performance of their 12-month-old footwear (2 patients) or to test the new prescribed footwear before reimbursement (9 patients).

Clinical and anthropometric data of the patients are reported in Table 4. Patients' classification as for risk level was done as in [3]. VPT and DNI were measured as in [20].

All patients but one, who suffered from poliomyelitis consequences, had off-the-shelf recommended shoes, in

Table 3. PP thresholds within the four foot regions, for all the expected-to-be-used Pedar Wide Insoles and for the three levels of risk.

\begin{tabular}{ccccc}
\hline & RF (kPa) & MF (kPa) & FF (kPa) & TO (kPa) \\
\hline Risk Level 0 - & & & & \\
UW & 230 & 100 & 270 & 160 \\
VW & 200 & 90 & 240 & 150 \\
WW & 180 & 80 & 220 & 130 \\
XW & 160 & 70 & 200 & 120 \\
YW & 150 & 70 & 180 & 110 \\
Risk Level 2 & & & & \\
UW & 210 & 60 & 240 & 140 \\
VW & 180 & 60 & 220 & 130 \\
WW & 160 & 60 & 200 & 120 \\
XW & 140 & 60 & 180 & 110 \\
YW & 130 & 60 & 160 & 100 \\
Risk Level 3 & 130 & 60 & 140 & 90 \\
UW & 180 & 60 & 220 & 130 \\
VW & 160 & 60 & 190 & 120 \\
WW & 140 & 60 & 180 & 100 \\
XW & 130 & 60 & 160 & 100 \\
YW & 120 & 60 & & \\
\hline
\end{tabular}

*New Diabetic footwear test protocol.
Table 4. Clinical and anthropometric data of the 11 Diabetic neuropathic patients examined during the preliminary footwear test session.

\begin{tabular}{cccccccc}
\hline & $\begin{array}{c}\text { Age } \\
\text { (years) }\end{array}$ & $\begin{array}{c}\text { Height } \\
(\mathrm{cm})\end{array}$ & $\begin{array}{c}\text { Body } \\
\text { mass } \\
(\mathrm{kg})\end{array}$ & $\begin{array}{c}\text { BMI } \\
\left(\mathrm{kg} / \mathrm{m}^{2}\right)\end{array}$ & VPT & DNI & $\begin{array}{c}\text { Risk } \\
\text { class }\end{array}$ \\
\hline P1 & 74 & 155 & 80 & 33 & 27 & 6.0 & 2 \\
P2 & 58 & 155 & 89 & 37 & 22 & 5.5 & 2 \\
P3 & 75 & 155 & 68 & 27 & 50 & 6.0 & 2 \\
P4 & 56 & 166 & 90 & 33 & 30 & 5.0 & 2 \\
P5 & 70 & 155 & 59 & 25 & 40 & 6.0 & 2 \\
P6 & 74 & 168 & 72 & 26 & 40 & 6.0 & 2 \\
P7 & 74 & 160 & 74 & 29 & 50 & 6.0 & 3 \\
P8 & 74 & 178 & 87 & 27 & 50 & 6.0 & 3 \\
P9 & 72 & 157 & 73 & 30 & 40 & 4.0 & 3 \\
P10 & 74 & 170 & 78 & 27 & 50 & 6.0 & 3 \\
P11 & 71 & 177 & 99 & 32 & 50 & 6.0 & 3 \\
\hline
\end{tabular}

some cases slightly adapted to the patient's needs, and custom-made insoles, usually obtained by a plaster mold of the patient's footprint while standing barefoot in the upright position. For those patients already treated at the Authors' Hospital, footwear prescriptions had been made on the basis of a consolidated procedure [3,23].

15 minutes were sufficient, on average, to perform the whole test protocol and to prepare the test report. In one case 10 additional minutes were required since the patient, suffering from the consequences of poliomyelitis, had the right foot much smaller than the left foot, which required a special adaptation of the whole test equipment and of processing procedures. All patients reacted positively to the test. The operator was able to prepare a clear report for each patient including over-threshold PP and GL deviations, to interpret it and to transfer the main suggestions to the clinician for the final decision.

On the basis of the above reports, of each patient's co-morbidities, clinical history and special needs, 4 new prescribed footwear did not pass the test; they had PP over thresholds (3 out of 4) and excessive GL medial and forward deviation (4 out of 4). In all cases specific requirements and recommendations were added to the previous prescription to better address the requested modifications. The remaining 5 new prescribed footwear passed the test; however, minor changes or integrations were asked to the orthopedic workshop before reimbursement: in 3 cases a replacement of the off-the-shelf shoes was recommended since they were too much interfering with the patient's gait, thus leading to an excessive forefoot pronation or an excessive GL forward shift; 
in 2 cases a softer and thicker damping cover was requested to be added to the custom-made insoles; in 1 case a silicone support for the toes was asked as an additional aid. The two 12-month-old footwear proved to be no more effective or even dangerous for the patients; a new prescription was done in both cases, with specific requirements for offloading of midfoot and toes (1 case), very high offloading of midfoot (1), control of midfoot and forefoot pronation (1 case).

As an example, the electronic copy of the paper sheet for test report is shown in Figure 4. It is referred to a footwear test with negative result.

\subsection{Validation Results}

For each patient and each foot region Table 5 indicates the baropodometric parameters which were found to be higher than the reference values. PP and GL alterations are reported together in the first row (in bold), and represent the only data the operator took into consideration during the footwear test; worth to remember, PP values were judged with respect to the specific PP thresholds. Max F, PTI, FTI and \% Stance were not made available to the operator; their alterations had been successively highlighted by comparing them with the healthy volunteers reference database.

As shown in the table, PP and GL alterations were well related to alterations of the other relevant parameters. On these preliminary data set, sensitivity was equal to $96.5 \%$, specificity was equal to $51.6 \%$. Only for two parameters out of the overall 88 reported in the table, PP and GL failed in detecting gait alterations, namely under the right toes of Patient 1 and under the left forefoot of Patient 6 (patient with poliomyelitis consequences).

Detailed analysis of averaged regional baropodometric parameters under the two testing conditions (i.e. with reference shoes and with prescribed footwear) is beyond

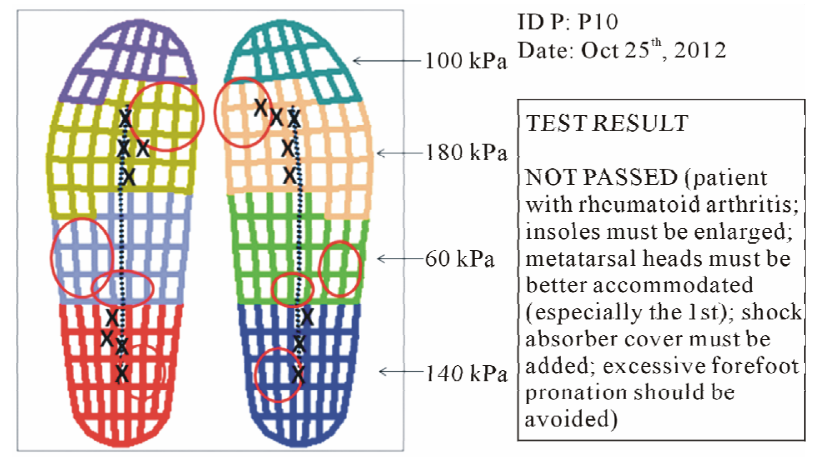

Legend $\bigcirc$ indicates pressure over threshold

$$
X \text { indicates gait line deviation }
$$

Figure 4. Instrumental test report of Patient P10. Over-threshold PPs were found in the rearfoot and in the midfoot, and under the first metatarsal area. GL was significantly shifted forward on the rearfoot and medially on the forefoot.
Table 5. Alterations of gait parameters with respect to healthy volunteers reference databases. A red cross indicates a "false positive" i.e. alteration of PP or GL not related to other gait alterations. A red box indicates a "false negative", i.e. alteration of other gait parameters which was not detected through PP or GL alterations.

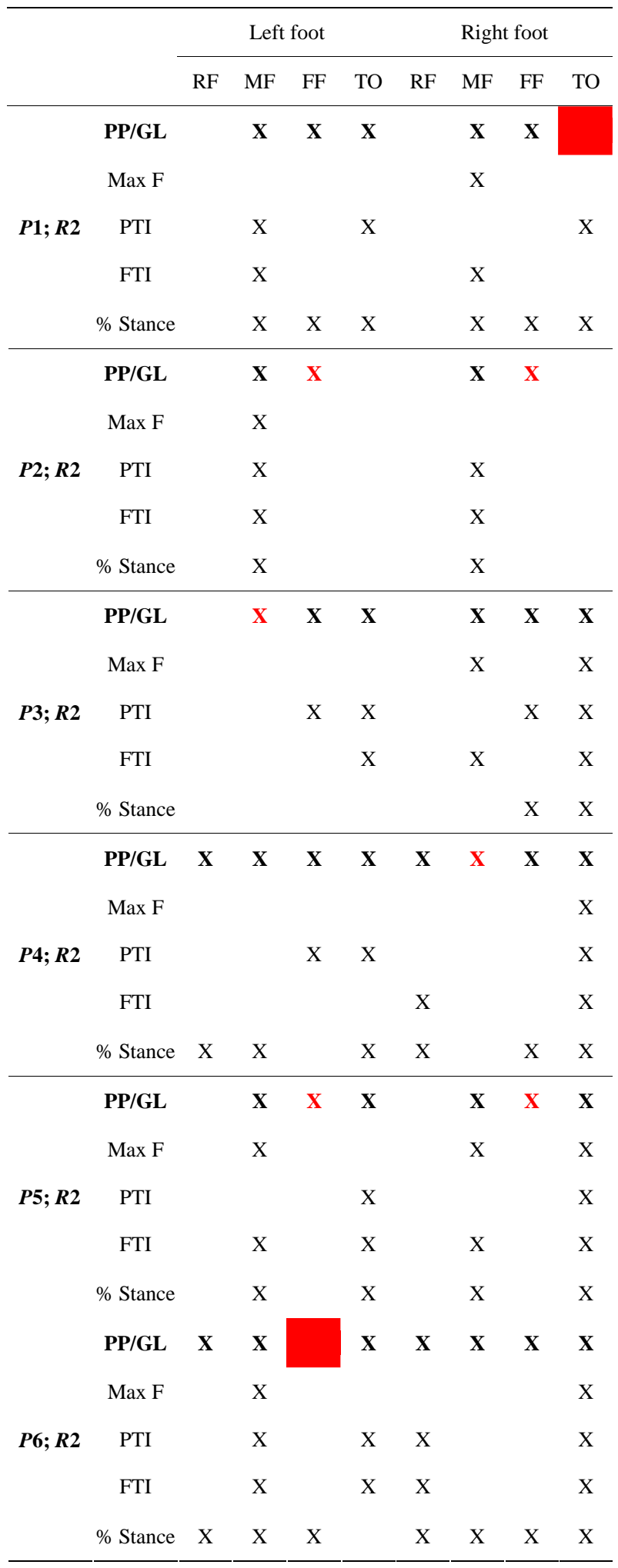




\section{Continued}

\begin{tabular}{|c|c|c|c|c|c|c|c|c|c|}
\hline \multirow{5}{*}{ P7; R3 } & PP/GL & & $\mathbf{X}$ & $\mathrm{X}$ & $\mathbf{X}$ & & $\mathbf{X}$ & $\mathbf{X}$ & $\mathbf{X}$ \\
\hline & Max F & & $\mathrm{X}$ & & $\mathrm{X}$ & & $\mathrm{X}$ & & \\
\hline & PTI & & & & $\mathrm{X}$ & & & & $\mathrm{X}$ \\
\hline & FTI & & $\mathrm{X}$ & & $\mathrm{X}$ & & $\mathrm{X}$ & & $\mathrm{X}$ \\
\hline & $\%$ Stance & & $\mathrm{X}$ & & $\mathrm{X}$ & & $\mathrm{X}$ & $\mathrm{X}$ & $\mathrm{X}$ \\
\hline \multirow{5}{*}{$P 8 ; R 3$} & PP/GL & $\mathbf{X}$ & $\mathbf{X}$ & $\mathrm{X}$ & $\mathrm{X}$ & $\mathbf{X}$ & $\mathbf{X}$ & $\mathrm{X}$ & \\
\hline & Max F & & $\mathrm{X}$ & & & & $\mathrm{X}$ & & \\
\hline & PTI & & $\mathrm{X}$ & & & & $\mathrm{X}$ & & \\
\hline & FTI & & $\mathrm{X}$ & & & & $\mathrm{X}$ & & \\
\hline & \% Stance & $\mathrm{X}$ & $\mathrm{X}$ & & & $\mathrm{X}$ & $\mathrm{X}$ & $\mathrm{X}$ & \\
\hline \multirow{5}{*}{$P 9 ; R 3$} & PP/GL & & $\mathbf{X}$ & $\mathrm{X}$ & $\mathrm{X}$ & $\mathbf{X}$ & $\mathbf{X}$ & $\mathrm{X}$ & $\mathrm{X}$ \\
\hline & Max F & & & & & & & & $\mathrm{X}$ \\
\hline & PTI & & $\mathrm{X}$ & $\mathrm{X}$ & $\mathrm{X}$ & $\mathrm{X}$ & $\mathrm{X}$ & & $\mathrm{X}$ \\
\hline & FTI & & $\mathrm{X}$ & & $\mathrm{X}$ & $\mathrm{X}$ & & & $\mathrm{X}$ \\
\hline & \% Stance & & $\mathrm{X}$ & $\mathrm{X}$ & $\mathrm{X}$ & & & & $\mathrm{X}$ \\
\hline \multirow{5}{*}{$P 10 ; R 3$} & PP/GL & $\mathrm{X}$ & $\mathbf{X}$ & $\mathrm{X}$ & & $\mathbf{X}$ & $\mathrm{X}$ & $\mathbf{X}$ & \\
\hline & Max F & & $\mathrm{X}$ & & & & $\mathrm{X}$ & & \\
\hline & PTI & & $\mathrm{X}$ & $\mathrm{X}$ & & & & $\mathrm{X}$ & \\
\hline & FTI & & $\mathrm{X}$ & & & & $\mathrm{X}$ & & \\
\hline & \% Stance & & $\mathrm{X}$ & $\mathrm{X}$ & & & $\mathrm{X}$ & & \\
\hline \multirow{5}{*}{ P11; R3 } & PP/GL & $\mathbf{X}$ & $\mathrm{X}$ & $\mathrm{X}$ & $\mathrm{X}$ & $\mathbf{X}$ & $\mathbf{X}$ & $\mathbf{X}$ & $\mathrm{X}$ \\
\hline & Max F & & $\mathrm{X}$ & & & & $\mathrm{X}$ & & \\
\hline & PTI & & $\mathrm{X}$ & & & $\mathrm{X}$ & $\mathrm{X}$ & & $\mathrm{X}$ \\
\hline & FTI & & $\mathrm{X}$ & & & $\mathrm{X}$ & $\mathrm{X}$ & & $\mathrm{X}$ \\
\hline & \% Stance & & $\mathrm{X}$ & $\mathrm{X}$ & & & & & \\
\hline
\end{tabular}

the scope of this study and may be of limited validity due the poor number of patients and the high variability of patients' conditions and footwear. Only the main results of a basic statistical analysis are here reported with respect to PTI, since they may be helpful for a preliminary validation of the test. Figure 5 shows mean values, standard deviations and statistically significant differences (Student's t-test, $\mathrm{p}<0.005$ ) of PTIs calculated with respect to the four foot regions in the three groups i.e. the 20 healthy volunteers walking with the reference shoes (group $\mathrm{H}$ ), the 11 Diabetic patients walking with the same reference shoes as healthy volunteers (group PR), the 11 patients walking with their prescribed footwear (group PF). Data proved that PTI distribution in group

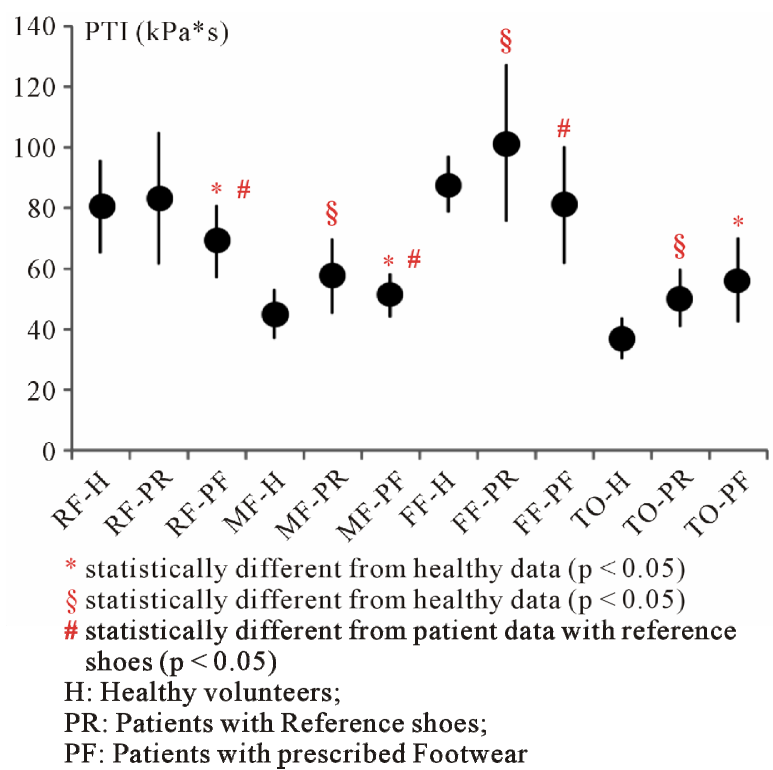

Figure 5. PTI mean values \pm sd, associated with the four foot regions ( $\mathrm{RF}, \mathrm{MF}, \mathrm{FF}$ and $\mathrm{TO}$ ) in three groups: healthy volunteers (group $\mathrm{H}$ ), patients with reference shoes (group PR), patients with prescribed footwear (group PF). Healthy volunteers were examined with the same reference shoes. Healthy reference data were averaged over 40 feet, patient data over 22 feet in both walking conditions. Differences were investigated by using the student's t-test $(\mathrm{p}<0.05)$.

PR was different from group $\mathrm{H}$, thus confirming that the measurement equipment and protocol can be suitable to highlight differences between healthy volunteers and Diabetic neuropathic patients. Furthermore, differences were evident between performance of Diabetic neuropathic patients with reference shoes and with prescribed footwear, which confirms that the latter may modify the gait pattern: PTI reduction in all regions but the toes, in fact, can be interpreted as a sign of the desired offloading effect due to the synergic action of proper shoes ad insoles. Reasonably, however, averaged data are confounded and statistical meaning partly masked by the inclusion of all tested footwear, either accepted or refused.

\section{DISCUSSION}

Learning from personal and other valuable researchers' experience in the field of research applied to the biomechanics of the Diabetic neuropathic foot, Authors made here an attempt to design, build, implement and validate an instrumental protocol for supporting clinical decision with respect to prescribed Diabetic footwear. Several factors played a key-role in the entire process: 1) evidence from the literature with respect to the role of plantar peak pressure as a reliable indicator of risky tissue damage under the sole of the foot of Diabetic neuropathic patients; 2) clinical evidence that insole plantar 
pressures below a certain threshold are strongly related to the maintenance of healthy or healed status of the foot; 3) experimental findings that further specificity of peak pressure thresholds may help in better discriminate performance and appropriateness of prescribed footwear; 4) proper knowledge and use of the adopted insole pressure measurement system so as to design a test protocol specifically tailored to exploit the system peculiarities and to cope with its limitations; 5) knowledge of the main needs of an outpatient service for Diabetic foot to deliver adequate clinical decisions with respect to prescribed footwear acceptance; 6) experience in measuring foot-ground interaction under various conditions and knowledge of the relevant changes in the outcomes of the main parameters of interest.

The following approach was then followed:

- A set of 20 healthy volunteers, homogeneous with the expected Diabetic population as for age, BMI and walking speed was recruited and examined while walking with modified semi-rigid shoes (smoothed Terapes).

- Based on the technical features of the used wearable pressure measurement system (Pedar-X Insole System), a set of insoles was defined (wide insoles in the range UW-YW, i.e. from EU size 36 to 45); four regions were identified and adapted to each insole and corresponding foot sizes to investigate rearfoot, midfoot, forefoot and toes; averaged reference values were calculated for main baropodometric parameters i.e. PP, normalized Max F, PTI, normalized FTI and $\%$ Stance (at least 20 footprints were processed for each volunteer, for left and right foot separately).

- Based on some geometrical data-i.e. the \% increase of sensor area with the increase of the insole sizeand the assumption of $10 \%$ of PP variation between two successive levels of ulceration risk, conservative factors have been taken into account and PP threshold maps have been created for each foot size and each of the three levels of risk; reference PP threshold maps and reference GL were created and pre-printed on paper sheets to promptly assess prescribed footwear in the outpatient service context.

- During the first validation session, the instrumental test protocol was applied to 11 Diabetic patients; data were acquired under two walking conditions, i.e. with the reference shoes and with each patient's own prescribed footwear; only PP and GL from the latter were compared with the established thresholds and used to support the final clinical decision; special care was taken not to refer to the maximum peak pressure maps directly available as a first software outcome: rather, the step analysis process was performed so as to obtain the map of the averaged peak pressures, average being referred to at least 20 footprints for each foot.

Preliminary results were encouraging. The instrumental test protocol was feasible, and well accepted by patients and operators. As a secondary interesting outcome, patients were interested in understanding the clinical meaning of the measurements, which was interpreted as a possible means to further educate and motivate them in wearing the proper footwear. As for the involved human resources, one week of training had been necessary to instruct the operators with basic knowledge of plantar pressure measurement and of the specific measurement system and protocol, and to allow them to correctly interpret the main outcomes, to report them on the structured pre-printed report, and to finally transfer the main findings and suggestions from the test to the clinician in charge for the prescribed footwear approval. Until now, the instrumental system management, as well as the data processing, have been kept under the direct supervision of the Authors, but the routine application of the test at the Hospital outpatient service, which will be hopefully implemented in the next future, will allow the preparation of more, qualified operators. One dedicated operator and 15 additional minutes were requested, on average, to add the instrumental test to the usual clinical examination and visual footwear inspection.

Contribution of the instrumental test to the clinical decision was of great relevance during the preliminary validation session: 1) 4 new footwear did not receive approval due to the PP values over the established thresholds; in two of the 4 cases the visual inspection from the expert clinician had already raised doubts about the appropriateness of the insoles, which were confirmed by the instrumental test; in the other two cases, the negative decision was mainly based on the instrumental test results associated with patient's comorbidities and very high level of risk; 2) 5 new footwear were approved but minor improvements were asked before reimbursement which would have not been requested without the instrumental test; 3) 2 old footwear were proved no more effective or even dangerous after 12 months of use; likely, a new prescription would have been done in any case, due to the Italian Healthcare Service Guidelines; however, indeed innovative for the Authors' outpatient service, the instrumental test allowed to add further specifications to the usual footwear prescription.

As reasonable, the true effectiveness of the proposed instrumental test could not been quantified yet; even, the modified prescriptions could not be tested with the same protocol to check for the proper implementation of the suggestions; however, as a first feedback, after 4 months none of the 11 examined patients showed plantar ulceration or sign of tissue damage. Furthermore, the analysis of the entire set of the relevant baropodometric parameters highlighted that the test, based on the PP specific 
thresholds and the reference GL deviations, was highly sensitive (96.5\%). Thus it can be considered safe and suitable for its inclusion in the clinical routine of the outpatient service, even though it may be considered too much conservative (specificity $=51.6 \%$ ).

Even though they have not a general validity, and a wider population should be examined and monitored, the baropodometric outcomes averaged over these preliminary 11 patients were encouraging, since they showed the potential of the whole measurement equipment and protocol in detecting the main alterations of the Diabetic neuropathic patients' gait, and the main effects due to the use of the preventive footwear.

The hypotheses and the assumptions which formed the basement for the design of the proposed test protocol are quite new: 1) reference healthy data should only be extracted from healthy volunteers who did not show any sign of tissue damage, thus demonstrating that the mechanical stress applied during the regular daily activities was appropriate for the foot; 2) healthy data acquisition under conditions as close as possible to those of the expected Diabetic population may allow to reliably use not only reference peak pressures or force data, but also pressure-time integrals, force-time integrals, and \%stance phases; 3) PP thresholds, while keeping in mind the upper threshold of $200 \mathrm{kPa}$ as reported in the qualified literature, should be tailored to the specific technical features of the measurement systems and to the specific level of risk of ulceration: while the former requirement can be managed on the basis of sensor geometry, the latter can be approached with assumptions related to the higher fragility of the Diabetic foot skin and to some experimental observations.

\section{Test Protocol Applicability and Limitations}

The proposed protocol proved to be feasible, safe, highly sensitive and at least useful in supporting the clinical decision with respect to prescribed footwear aimed at preventing ulceration or recurrence of ulceration in Diabetic neuropathic patients. While the overall methodology may have a general validity and may be applied in association with other insole measurement systems, the reference data and the established PP thresholds and GL pattern can be considered valid only in case a calibrated Pedar-X Insole measurement system is used, in association with Pedar wide insoles in the range UW-YW.

To obtain evidence of the protocol effectiveness a wider population needs to be examined and monitored during time; hopefully, the implementation of the same protocol under comparable conditions at the Authors' but also at other Hospital outpatient services will help in better investigating not only the crucial issue of clinical effectiveness but also the important aspect of cost-effectiveness and optimization of healthcare resources.
One strong limitation of the protocol are the assumptions done to arbitrarily decrease of $10 \%$ the PP threshold for each increasing level of risk, and to arbitrarily limit the PP midfoot threshold at $60 \mathrm{kPa}$ for risk 2 and 3 (high and very high risk) independently on foot size. These thresholds, always below the recommended threshold of $200 \mathrm{kPa}$ [14], proved to be highly sensitive in the preliminary test session when used in conjunction with reference GL (96.5\%) but, at the same time, they showed a moderate specificity (51.6\%). Reasonably, the PP thresholds will deserve deeper investigation in future studies.

Another important "limitation", for those who are in search of completely automated assessment protocol, is the essential contribution of operator and clinician's expertise and knowledge of patient's clinical history and concurrent pathologies in reaching the final decision. Some aspects will likely be automated in future processing tools and very simple expert-system; however, Authors' strongly believe in the high added value of human independent decision and numbers interpretation.

\section{CONCLUSIONS}

Aiming at improving the foot care of Diabetic neuropathic patients, a simple, reliable, fast and easy-to-use instrumental test protocol has been here proposed to support clinical decision with respect to the approval, the rejection or the modification of prescribed footwear for ulcer prevention. Major strengths of the protocol, quite innovative with respect to the instrumental test scenario, are: the adaptation of the relevant indicators and threshold values to the technical features of the used pressure measurement system and to the specific level of risk; the identification of only four major foot regions and only two indicators, i.e. PP threshold and GL pattern, for the prompt use of outcomes; the compliance with the specific needs of an outpatient Diabetic foot test service.

Encouraging feedback came from the preliminary validation session: the protocol was well accepted by patients and operators, fast and easy to be used; properly integrated with clinical and visual inspection data, it seems to effectively contribute to the improvement of footwear prescription, test and monitoring during their lifetime (none of the 11 tested patients developed ulcers or tissue damage in the successive 4 months); it proved to be sensitive and valuable for the Diabetic foot care, even though further studies are needed to improve its specificity. The protocol itself, hereby reported in detail, has a general validity; regardless of the test equipment and software used, it is only recommended to never use the maximum PPs which are promptly made available by all commercial systems but rather to compare thresholds with PP averaged over a proper number of steps (operators must be properly trained about this specific "pitfall”). 
However, the hereby delivered specific PP thresholds and reference data maintain their validity only when the test is performed under the same instrumental conditions, i.e. with a calibrated Pedar Insole System and with the wide Insoles in the range UW-YW (EU size 36 - 45).

\section{ACKNOWLEDGEMENTS}

Authors would like to thank all the operators of the Authors' University Hospital who rendered the study feasible, and all patients and healthy volunteers who kindly agreed to participate in the study.

\section{REFERENCES}

[1] Apelqvist, J., Bakker, K., van Houtum, W.H., NabuursFranssen, M.H., Schaper, N.C. and International Working Group on the Diabetic Foot (2000) International consensus and practical guidelines on the management and the prevention of the diabetic foot. Diabetes/Metabolism Research and Reviews, 16, S84-S92. doi:10.1002/1520-7560(200009/10)16:1+<::AID-DMRR 113>3.0.CO;2-S

[2] Bakker, K., Apelqvist, J., Schaper, N.C. and International Working Group on Diabetic Foot Editorial Board (2012) Practical guidelines on the management and prevention of the diabetic foot 2011. Diabetes/Metabolism Research and Reviews, 28, 225-31. doi:10.1002/dmrr.2253

[3] Uccioli, L. and Giacomozzi, C. (2009) Biomechanics and choosing footwear for the diabetic foot. The Diabetic Foot Journal, 12, 165-175.

[4] Waaijman, R., Keukenkamp, R., De Haart, M., Polomski, W.P., Nollet, F. and Bus, S.A. (19 February 2013) Adherence to wearing prescription custom-made footwear in patients with Diabetes at high risk for plantar foot ulceration. Diabetes Care, Published Online.

[5] Paton, J.S., Stenhouse, E.A., Bruce, G., Zahra, D. and Jones, R.B. (2012) A comparison of customised and prefabricated insoles to reduce risk factors for neuropathic diabetic foot ulceration: A participant-blinded randomised controlled trial. Journal of Foot and Ankle Research, 5, 31. doi:10.1186/1757-1146-5-31

[6] Ibrahim, M., El Hilaly, R., Taher, M. and Morsy, A. (2013) A pilot study to assess the effectiveness of orthotic insoles on the reduction of plantar soft tissue strain. Clinical Biomechanics, 28, 68-72. doi:10.1016/j.clinbiomech.2012.09.003

[7] Rouhani, H., Favre, J., Crevoisier, X. and Aminian, K. (2011) Ambulatory measurement of ankle kinetics for clinical applications. Journal of Biomechanics, 44, 27122718. doi:10.1016/j.jbiomech.2011.07.021

[8] Bus, S.A, Haspels, R. and Busch-Westbroek, T.E. (2011) Evaluation and optimization of therapeutic footwear for neuropathic diabetic foot patients using in-shoe plantar pressure analysis. Diabetes Care, 34, 1595-600. doi:10.2337/dc10-2206

[9] Spooner, S.K., Smith, D.K. and Kirby, K.A. (2010) Inshoe pressure measurement and foot orthosis research: A giant leap forward or a step too far? Journal of the Am- erican Podiatric Medical Association, 100, 518-529.

[10] Bennetts, C.J., Owings, T.M., Erdemir, A., Botek, G. and Cavanagh, P.R. (2013) Clustering and classification of regional peak plantar pressures of diabetic feet. Journal of Biomechanics, 46, 19-25. doi:10.1016/j.jbiomech.2012.09.007

[11] Giacomozzi, C. and Martelli, F. (2006) Peak pressure curve: An effective parameter for early detection of foot functional impairments in diabetic patients. Gait Posture, 23, 464-470.

[12] Macfarlane, R.M. and Jeffcoate, W.J. (1997) Factors contributing to the presentation of diabetic foot ulcers. Diabetic Medicine, 14, 867-870. doi:10.1002/(SICI)1096-9136(199710)14:10<867::AIDDIA475>3.0.CO;2-L

[13] Veves, A., Murray, H.J., Young, M.J. and Boulton, A.J. (1992) The risk of foot ulceration in diabetic patients with high foot pressure: a prospective study. Diabetologia, 35, 660-663. doi:10.1007/BF00400259

[14] Owings, T.M., Apelqvist, J., Stenstrom, A., Becker, M., Bus, S.A., Kalpen, A., Ulbrecht, J.S. and Cavanagh, P.R. (2009) Plantar pressures in diabetic patients with foot ulcers which have remained healed. Diabetic Medicine, 26, 1141-1146. doi:10.1111/j.1464-5491.2009.02835.x

[15] Delzell, E. (2011) The role of rocker soles in reducing ulcer risk.

http://lowerextremityreview.com/article/the-role-of-rocke r-soles-in-reducing-ulcer-risk

[16] Giacomozzi, C. (2010) Appropriateness of plantar pressure measurement devices: A comparative technical assessment. Gait \& Posture, 32, 141-144. doi:10.1016/j.gaitpost.2010.03.014

[17] Giacomozzi, C., Keijsers, N., Pataky, T. and Rosenbaum, D. (2012) International scientific consensus on medical plantar pressure measurement devices: Technical requirements and performance. Annali dell'Istituto Superiore di Sanita, 48, 259-271. doi:10.4415/ANN $1203 \quad 06$

[18] Giacomozzi, C. (2010) Hardware performance assessment recommendations and tools for baropodometric sensor systems. Annali dell'Istituto Superiore di Sanita, 46, 158-167.

[19] Giacomozzi, C. (2011) Potentialities and criticalities of plantar pressure measurements in the study of foot biomechanics: Devices, methodologies and applications. In: Klika, V., Ed., Biomechanics in Applications, Chapter 11, InTech Publication. doi:10.5772/23464 http://www.intechopen.com/books/biomechanics-in-appli cations/potentialities-and-criticalities-of-plantar-pressuremeasurements-in-the-study-of-foot-biomechanics

[20] Giacomozzi, C., Caselli, A., Macellari, V., Giurato, L., Lardieri, L. and Uccioli, L. (2002) Walking strategy in diabetic patients with peripheral neuropathy. Diabetes Care, 25, 1451-1457. doi:10.2337/diacare.25.8.1451

[21] Arts, M.L.J. and Bus, S.A. (2011) Twelve steps per foot are recommended for valid and reliable in-shoe plantar pressure data in neuropathic diabetic patients wearing custom made footwear. Clinical Biomechanics, 26, 880884. doi:10.1016/j.clinbiomech.2011.05.001 
[22] Giacomozzi, C., Benedetti, M.G., Leardini, A., Macellari, V. and Giannini, S. (2006) Gait analysis with an integrated system for functional assessment of talocalcaneal coalition. Journal of the American Podiatric Medical Association, 96, 107-115.
[23] Rutger Dahmen, R., Haspels, R., Koomen, B. and Hoeksma, A.F., (2001) Therapeutic footwear for the neuropathic foot: An algorithm. Diabetes Care, 24, 705-709. doi:10.2337/diacare.24.4.705 